\title{
Enhanced Epithelial-to-Mesenchymal Transition Associated with Lysosome Dysfunction in Podocytes: Role of p62/ Sequestosome 1 as a Signaling Hub
}

\author{
Guangbi Lia Cai-Xia Lia Min Xia ${ }^{a}$ Joseph K. Ritter ${ }^{\mathrm{a}}$ Todd W. B. Gehr \\ Krishna Boinia Pin-Lan Li ${ }^{a}$ \\ Department of a Pharmacology and Toxicology and ${ }^{\mathrm{b} I n t e r n a l ~ M e d i c i n e, ~ S c h o o l ~ o f ~ M e d i c i n e, ~ V i r g i n i a ~}$ \\ Commonwealth University, Richmond, VA, USA
}

\section{Key Words}

Podocyte transdifferentiation • Glomerulosclerosis • Selective autophagy • Lysosomal enzymes - Ubiquitin binding protein

\begin{abstract}
Background: Autophagy is of importance in the regulation of cell differentiation and senescence in podocytes. It is possible that derangement of autophagy under different pathological conditions activates or enhances Epithelial-to-Mesenchymal Transition (EMT) in podocytes, resulting in glomerular sclerosis. To test this hypothesis, the present study produced lysosome dysfunction by inhibition of the vacuolar $\mathrm{H}^{+}$-ATPase (V-ATPase) to test whether deficiency of autophagic flux leads to enhancement of EMT in podocytes. Methods and Results: By Western blot and confocal analysis, lysosome inhibition using a V-ATPase inhibitor or its siRNA was found to markedly decreases the epithelial markers ( $\mathrm{P}$-cadherin and ZO-1) and increases the mesenchymal markers (FSP-1 and $\alpha-S M A)$. This enhancement was accompanied by deficient autophagic flux, as demonstrated by marked increases in LC3B-II and p62/Sequestosome 1. However, inhibition of autophagosome formation using spaudin-1 significantly attenuated both enhancement of EMT and deficiency of autophagic flux. To explore the mechanisms by which deficient autophagic flux enhances EMT, we tested the role of accumulated p62 as a signal hub in this process. Neither the nuclear factor erythroid 2related factor 2 (Nrf2) and nuclear kappa-light-chain-enhancer pathways of p62 contributed to enhanced EMT. However, inhibition of cyclin-dependent kinase 1 (CDK1) activity reduced the phosphorylation of p62 and enhanced EMT in podocytes similar to lysosome dysfunction. Conclusion: The lack of phosphorylated p62 leads to a faster exit from cell mitosis, enhanced EMT associated with lysosome dysfunction may be attributed to accumulation of p62 and associated reduction of p62 phosphorylation.
\end{abstract}

Copyright $\odot 2015$ S. Karger AG, Basel

Pin-Lan Li, M.D, Ph.D.

KARGER 125
Department of Pharmacology and Toxicology, School of Medicine Virginia Commonwealth University, 1220 East Broad Street, Richmond, VA 23298 (USA) Tel. +1(804) 828-4793, Fax +1(804) 828-4794, E-Mail pli@vcu.edu 


\section{Cellular Physiology Cell Physiol Biochem 2015;35:1773-1786

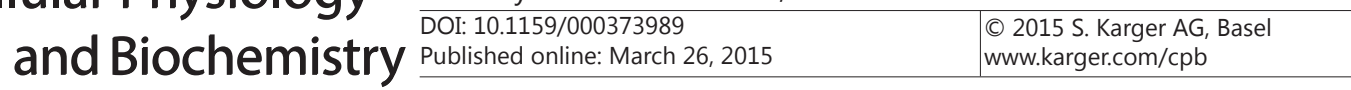 Li et al.: Lysosome Dysfunction and Podocyte Epithelial-to-Mesenchymal Transition}

\section{Introduction}

Recent studies have proposed two major hypotheses for the mechanism leading to podocyte injury under different pathological conditions. The first hypothesis emphasizes the importance of podocyte depletion resulting from apoptosis as a causative factor for the onset of proteinuria and glomerular sclerosis [1-3]. According to this hypothesis, the reduced podocyte number in glomeruli is attributed to the apoptotic death of these cells. Another hypothesis for podocyte injury proposes that injured podocytes obtain a motile ability facilitating their detachment from the glomerular basement membrane rather than apoptotic cell death, where podocytes are able to undergo an epithelial-to-mesenchymal transition (EMT) process when they are challenged by different injurious stimuli such as transforming growth factor- $\beta 1$ (TGF- $\beta 1$ ) $[4,5]$, high glucose $[4,6]$, homocysteine [7-11], and adriamycin [4]. It has been shown that the EMT process is characterized by loss of its epithelial features as indicated by reduced level of podocyte-derived proteins such as nephrin, P-cadherin (P-cad), and zonula occludens-1 (ZO-1) and by acquiring mesenchymal features such as increases in the expression of desmin, fibroblast-specific protein-1 (FSP-1), and $\alpha$-smooth muscle actin ( $\alpha$-SMA) [7]. This podocyte phenotype change may lead to disruption of its delicate architecture, impairing glomerular filtration membrane function and triggering glomerular injury and sclerosis $[12,13]$. However, it remains poorly understood how podocyte EMT is activated and regulated in response to different pathological stimuli.

In this regard, previous studies have demonstrated that podocyte differentiation and maturation are highly dependent upon normal autophagy $[2,13]$. Given that EMT occurs as transdifferentiation or like dedifferentiation, it is plausible that a deficient autophagy such as reduced autophagic flux contributes to the activation or enhancement of EMT. It is well known that autophagy is a cell survival mechanism responsible for the degradation of longlived or damaged proteins and excessive or dysfunctional cell organelles [12, 14, 15]. Under physiological conditions, autophagy functions in a continuous, reparative way to maintain normal cellular homeostasis. In addition to the formation of autophagosomes, autophagy also includes the autophagic flux consisting of the fusion of autophagosomes to lysosomes and the lysosomal enzymatic degradation of these autophagic substances. This autophagic flux is determined by lysosome function and therefore the normal lysosome function plays a critical role in maintenance of autophagic process, keeping podocytes in a differentiated and functional status. Indeed, we have recently reported that the regulation of lysosome function importantly contributes to autophagic flux or autophagy maturation in mouse podocytes and that lysosome dysfunction or injury due to derangement of its regulatory mechanisms resulted in deficiency of autophagic flux and consequent EMT $[13,16]$. It is now imperative to address how a deficient autophagic flux associated with lysosome dysfunction activates or enhances podocyte EMT.

In the present study, we performed a series of studies to test a hypothesis that lysosome dysfunction may induce podocyte EMT due to the accumulation of autophagosome, p62 aggregation, and activation of associated signaling pathways such as Nrf2-mediated redox sensing, NF-кB-dependent transcriptional regulation, and cyclin-dependent kinase 1 (CDK1)-mediated phosphorylation of p62. We first determined whether lysosome function inhibition by V-ATPase inhibitor and its siRNA induces EMT in podocytes by observations of changes in epithelial and mesenchymal markers. Then, we tested whether lysosome function inhibition leads to accumulation of autophagosome and aggregation of p62 in podocytes, a typical deficiency of autophagic flux, which may contribute to activation or enhancement of podocyte EMT. Finally, we went on to explore the mechanisms by which lysosome dysfunction leads to EMT by testing the role of p62-related signaling pathways. Our results demonstrate that p62 accumulation and associated reduction in phosphorylation of p62 may be a novel mechanism switching on EMT associated with lysosome dysfunction and autophagic flux deficiency in podocytes.

\section{KARGER}




\section{Cellular Physiology Cell Physiol Biochem 2015;35:1773-1786

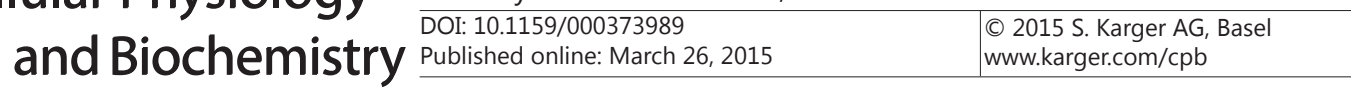 \\ Li et al.: Lysosome Dysfunction and Podocyte Epithelial-to-Mesenchymal Transition}

\section{Materials and Methods}

\section{Cell culture}

Conditionally immortalized mouse podocytes cell line, kindly provided by Dr. Klotman PE (Division of Nephrology, Department of Medicine, Mount Sinai School of Medicine, New York, NY, USA), were cultured on collagen I-coated flasks or plates in RPMI 1640 medium supplemented with recombinant mouse interferon $-\gamma$ at $33^{\circ} \mathrm{C}$. After differentiated at $37^{\circ} \mathrm{C}$ for 10 - 14 days without interferon- $\gamma$, podocytes were used for proposed experiments. The concentrations used for all protocols were decided based on our preliminary dose response experiments (from 2.5-20 ng/mL), which showed that $5 \mathrm{nM}$ Bafilomycin (Baf) had stable effects on activation of EMT in podocytes.

\section{Immunofluorescence microscopy}

Double-immunofluorescence staining was performed using cultured podocytes on cover slips. After fixation, the cells were incubated with rabbit anti-podocin 1: 200 (Sigma, St. Louis, MO, USA), which was followed by incubation with Alex-488- labeled donkey anti-rabbit secondary antibody. Then, goat antiFSP-1 (1:50 dilution), goat anti-ZO-1 (1:50 dilution) (Santa Cruz Biotechnology Inc, Santa Cruz, CA, USA), goat anti-P-cadherin (1:25 dilution), or mouse anti- $\alpha$-SMA (1:300 dilution) (R\&D system, Minneapolis, MN, USA) were added to the cell slides and then incubated overnight at $4^{\circ} \mathrm{C}$. After washing, the slides were incubated with corresponding the Alex-555-labeled secondary antibodies and then mounted and subjected to examinations using a confocal laser scanning microscope (Fluoview FV1000, Olympus, Japan). These double staining experiments were performed to observe the relationship between podocin production and EMT changes during Bafilomycin (Baf) incubation.

In addition to double staining and confocal microscopy, several groups of podocytes were used for quantitative analysis of expression of markers of EMT, autophagosome and lysosomes by fluorescence microscope. In these experiments, podocytes on cover slips were fixed in 4\% PFA for 20 minutes. After rinsed with phosphate-buffer saline (PBS), they were incubated with rabbit anti-FSP-1 (1:100, Abcam, Cambridge, MA, USA), rabbit anti-ZO-1 (1:50, Invitrogen, Camarillo, CA, USA), rabbit anti-P-cadherin (1:25), mouse anti- $\alpha$-SMA (1:300, R\&D system, Minneapolis, MN, USA) antibodies, rabbit anti-LC3B (Cell Signaling Technology, Beverly, MA, USA), or rat anti-LAMP1 (Novus Biologicals, St. Louis, MO, USA). After washing, the slides were incubated with the corresponding Alex-488-labeled secondary antibodies for $1 \mathrm{~h}$ at room temperature. After mounted with DAPI-containing mounting solution, the slides were observed under a fluorescence microscope and photos were taken and analyzed.

\section{Western blot analysis}

Western blot analysis was performed as described previously [7]. In brief, homogenates from cultured podocytes were prepared using sucrose buffer containing protease inhibitors. After boiling for $5 \mathrm{~min}$ at $95^{\circ} \mathrm{C}$ in a $5 \times$ loading buffer, $20 \mu \mathrm{g}$ of total proteins were subjected to SDS-PAGE, transferred onto a PVDF membrane and blocked by solution with dry milk. Then, the membrane was probed with primary antibodies of anti-ZO-1 (1:1000, Invitrogen), anti-P-cadherin (1: 1000, R\&D System), anti- $\alpha$-SMA (1:5000, R\&D System), anti-FSP-1 (1:1000, Abcam), anti-LC3B (1:1000, Cell Signaling Technology), anti-LAMP1 (1:1000, Novus Biologicals), mouse anti-p62 (1:5000, Abcam, Cambridge, MA, USA), rabbit anti-phospho-p62 (1:1000, Cell Signaling Technology, Beverly, MA, USA) or anti- $\beta$-actin (1:5000, Santa Cruz Biotechnology) overnight at 4 ${ }^{\circ} \mathrm{C}$ followed by incubation with horseradish peroxidase-labeled IgG (1:5000). The immunoreactive bands were detected by chemiluminescence methods and visualized on Kodak Omat X-ray films. Densitometric analysis of the images obtained from X-ray films was performed using the Image J software (NIH, Bethesda, MD, USA).

\section{siRNA transfection}

Vacuolar $\mathrm{H}^{+}$-ATPase siRNA and Nrf2 siRNA were purchased from (Qiagen, Valencia, CA, USA). The scrambled RNA (Qiagen, Valencia, CA, USA) was confirmed as non-silencing double-strand RNA and used as the control in the present study. Podocytes were serum starved for $12 \mathrm{~h}$ and then transfected with gp91phox small interfering RNA (siRNA) or scrambled siRNA using siLentFect Lipid Reagent (Bio-Rad, Hercules, CA, 


\section{Cellular Physiology Cell Physiol Biochem 2015;35:1773-1786

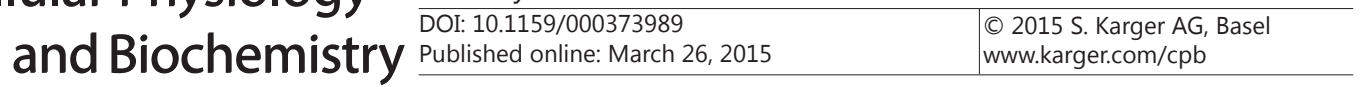 \\ Li et al.: Lysosome Dysfunction and Podocyte Epithelial-to-Mesenchymal Transition}

USA). After $24 \mathrm{~h}$ of incubation at $37^{\circ} \mathrm{C}$, the medium was changed, and $\mathrm{Baf}(5 \mathrm{nM})$ was added into the medium for indicated time span in different protocols.

\section{Statistical analysis}

All of the values are expressed as mean \pm SEM. Significant differences among multiple groups were examined using ANOVA followed by a Student-Newman-Keuls test. $\chi^{2}$ test was used to assess the significance of ratio and percentage data. $\mathrm{P}<0.05$ was considered statistically significant.

\section{Results}

Enhanced EMT by lysosome function inhibition in podocytes

To determine whether lysosome function inhibition has effects on EMT in podocytes, Western blot analysis was performed before and after inhibition of lysosome function by either bafilomycin, a well-known lysosome function inhibitor through its inhibitory effect on V-ATPase or siRNA of this enzyme. As shown in Fig. 1A and 1B, when podocytes were treated with bafilomycin, the epithelial markers P-cad and ZO-1 decreased significantly, while the mesenchymal markers FSP-1 and $\alpha$-SMA increased markedly. Similarly, V-H-ATPase siRNA transfection (siv-A) decreased P-cad and ZO-1 but increased FSP-1 and $\alpha$-SMA significantly in podocytes (Fig. 1C and 1D). It is clear that the ratio of epithelial markers vs. mesenchymal markers was significantly reduced during lysosome function inhibition, suggesting a large enhancement of EMT in podocytes.

\section{Confocal microscopy of EMT during lysosome function inhibition}

The effects of lysosome functional inhibition on EMT in podocytes were further detected using confocal microscopy. As shown in Fig. 2A, under basal condition podocytes were enriched with P-cadherin and Z0-1. When these podocytes were treated with bafilomycin, both P-cadherin and Z0-1 fluorescent staining were significantly reduced. As shown in

Fig. 1. Effects of lysosome function inhibition on EMT in podocytes. Podocytes were stimulated by bafilomycin $(5 \mu \mathrm{M})$ for 12 hours or transfected with v-Ca2+-ATPase siRNA (10 nM) for 24 hours. A. Representative gel documents showing the expression of ZO-1 and P-cadherin (P-cad) as epithelial markers and the expression of $\alpha$-SMA and FSP- 1 as mesenchymal markers in different groups. B. Summarized data showing the expression of ZO-1 and P-cadherin as epithelial markers and the expression of $\alpha$-SMA and FSP- 1 as mesenchymal markers, quantitated as a ratio of detected specific protein band vs. $\beta$-actin as loading control ( $n=3-5)$. C. Representative

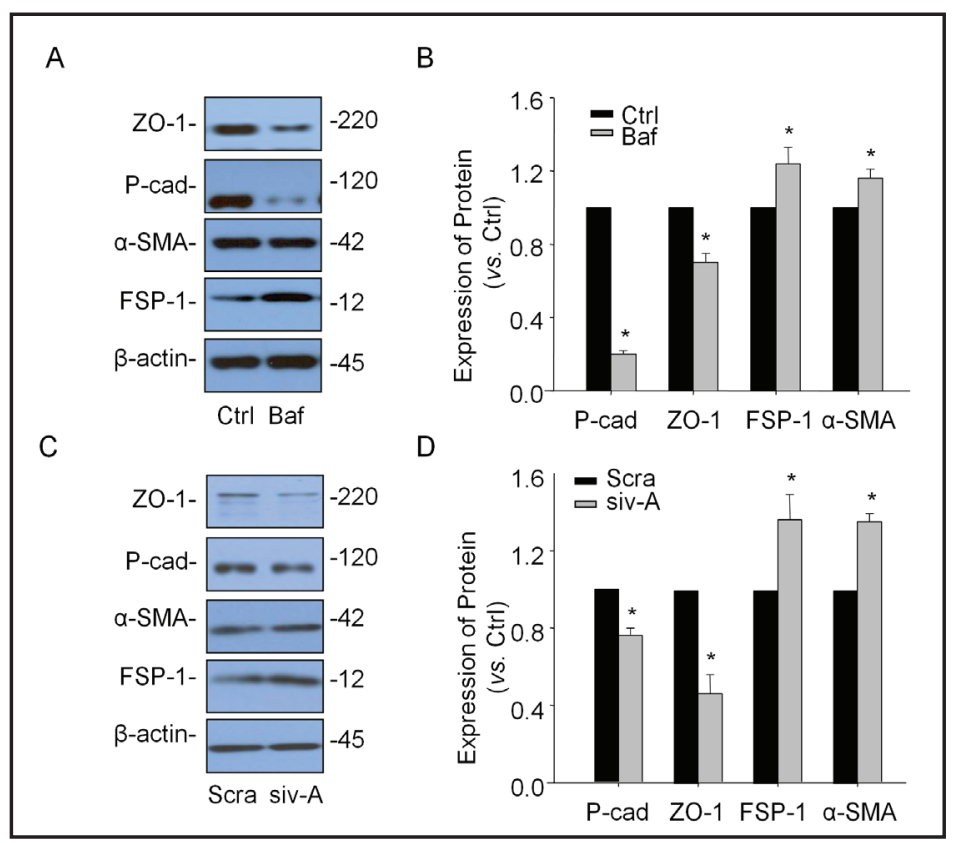
gel documents showing the expression of ZO-1 and P-cadherin as epithelial markers and the expression of $\alpha$-SMA and FSP-1 as mesenchymal markers in different groups. D. Summarized data showing the expression of ZO- 1 and P-cadherin as epithelial markers and the expression of $\alpha$-SMA and FSP-1 as mesenchymal markers, quantitated as a ratio of detected specific protein band vs. $\beta$-actin as loading control $(n=3-5) .{ }^{*} \mathrm{P}<0.05$ vs. Ctrl. Ctrl: Control, Baf: Bafilomycin, siv-A: V-H-ATPase siRNA, Scra: Scramble.

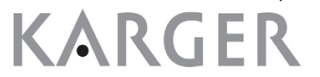




\section{Cellular Physiology Cell Physiol Biochem 2015;35:1773-1786 \begin{tabular}{ll|l} 
and Biochemistry & $\begin{array}{l}\text { DOI: 10.1159/000373989 } \\
\text { Publisnea onme. Vvarch 26, } 2015\end{array}$ & $\begin{array}{l}\text { ○ 2015 S. Karger AG, Basel } \\
\text { www.karger.com/cpb }\end{array}$ \\
\hline
\end{tabular}}

Li et al.: Lysosome Dysfunction and Podocyte Epithelial-to-Mesenchymal Transition

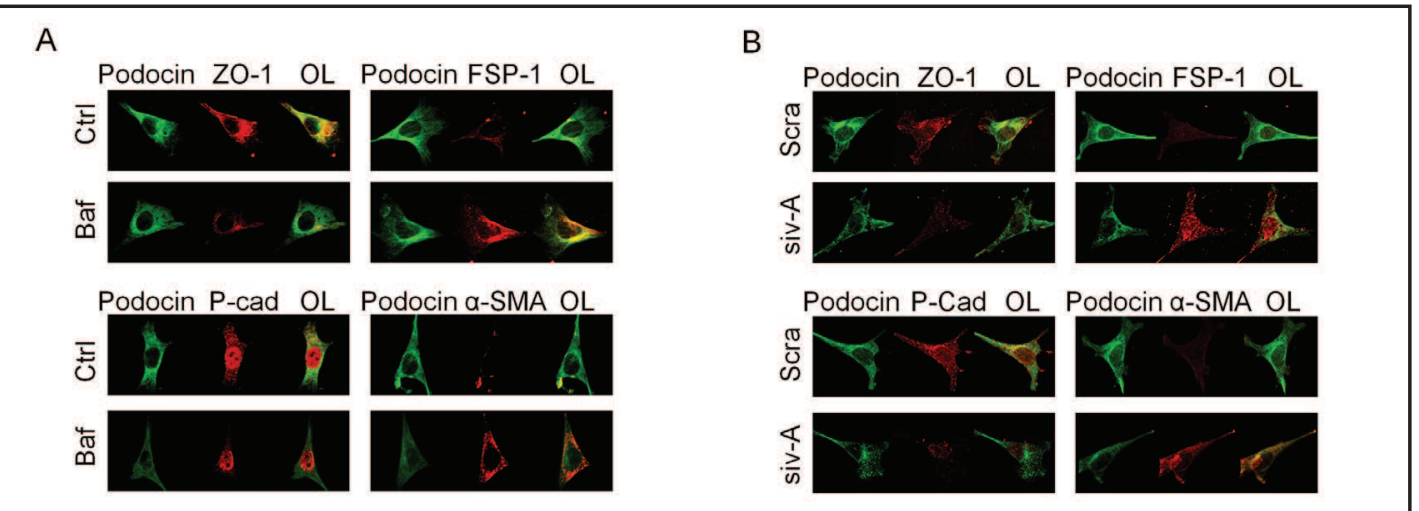

Fig. 2. Confocal microscopic of EMT changes by lysosome function inhibition. A. Images showing double-immunostained podocytes for epithelial markers, P-cadherin (p-cad) and Z0-1 (Alex555, red color) or mesenchymal markers, $\alpha$-SMA and FSP-1 (Alex555, red color) with podocyte marker, podocin (Alex488, green color) in different groups $(n=5)$. B. Images showing double-immunostained podocytes for epithelial markers, P-cadherin and Z0-1 (Alex555, red color) or mesenchymal markers, $\alpha$-SMA and FSP-1 (Alex555, red color) with podocyte marker, podocin (Alex488, green color) in different groups $(n=4)$. Ctrl: Control, Baf: Bafilomycin, siv-A: V-H-ATPase siRNA, OL: Overlaid.

overlaid images (OL in left panels of Fig. 2A), there were much less co-colocalized signals of podocin with ZO-1 or P-cadherin in bafilomycin-treated podocytes compared with control podocytes. In contrast, FSP-1 and $\alpha$-SMA staining increased by bafilomycin as shown by enhanced co-localization of podocin with both markers (right panels of Fig. 2A). In podocytes transfected with V-ATPase siRNA (siv-A), detected epithelial marker and mesenchymal marker stainings were similar to that shown in bafilomycin-treated podocytes, decreases in epithelial marker and increases in mesenchymal markers (Fig. 2B).

\section{Deficiency of autophagic flux during lysosome function inhibition}

Given the role of autophagy in podocyte differentiation, we tested whether enhanced EMT due to lysosome function inhibition is associated with deficient autophagy. Fig. 3A shows representative gel documents of Western blot using antibodies against LC3B, Lamp1 and p62. Under basal condition and co-treatment of podocytes with rapamycin (RPM, an autophagy inducer) or 7-keto (an autophagy stimulator), the protein levels of both LC3BII and p62 (two autophagosome markers) significantly increased by bafilomycin, while Lamp-1, a lysosome membrane marker was not changed. As shown in Fig. 3B, lysosome function inhibition mainly increased LC3II because the ratio of LC3B-II vs. LC3B-I was largely increased by bafilomycin, which were similar under control condition and with stimulation of autophagosome formation by RPM or 7-keto. The increased LC3BII level was accompanied by accumulation of p62 in podocytes (Fig. 3C). However, the Lamp-1 level was not significantly changed by bafilomycin (Fig. 3D). In addition, V-ATPase siRNA was also transfected in podocytes to determine whether lysosome function inhibition leads to deficient autophagic flux. It was found that lysosomal V-ATPase siRNA produced similar effect on autophagic flux to that made by bafilomycin, showing that both LC3B and p62 significantly increased in podocytes (data not shown).

\section{Attenuation of EMT by Sp-1 inhibition of autophagosome formation}

To further determine the role of autophagosome accumulation in podocyte EMT enhanced by lysosome function inhibition, we examined the effects of selective autophagosome formation inhibitor, Sp-1 on bafilomycin-enhanced EMT. As shown in Fig. 4A, a representative Western blot gel document showed that bafilomycin dramatically increased the LCB-II level. In the presence of Sp-1, the effects of bafilomycin on the LCBII level were largely weakened. As summarized in Fig. 4B, the ratio of LC3B-II vs. LC3B-I was significantly 


\section{Cellular Physiology Cell Physiol Biochem 2015;35:1773-1786

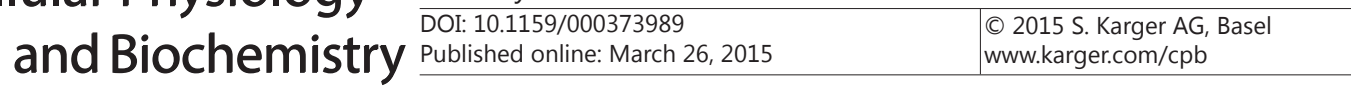

Fig. 3. Effects of lysosome function inhibitor on autophagy. Podocytes were cultured for 24 hrs with rapamycin (100 nM) or 7-keto cholesterol $(5 \mu \mathrm{g} / \mathrm{ml})$ in the absence or presence of bafilomycin A1 (10 $n M)$. A. Representative gel documents showing the expression of LC3B-I, LC3B-II, p62 as autophagosome markers and Lamp-1 as lysosome marker

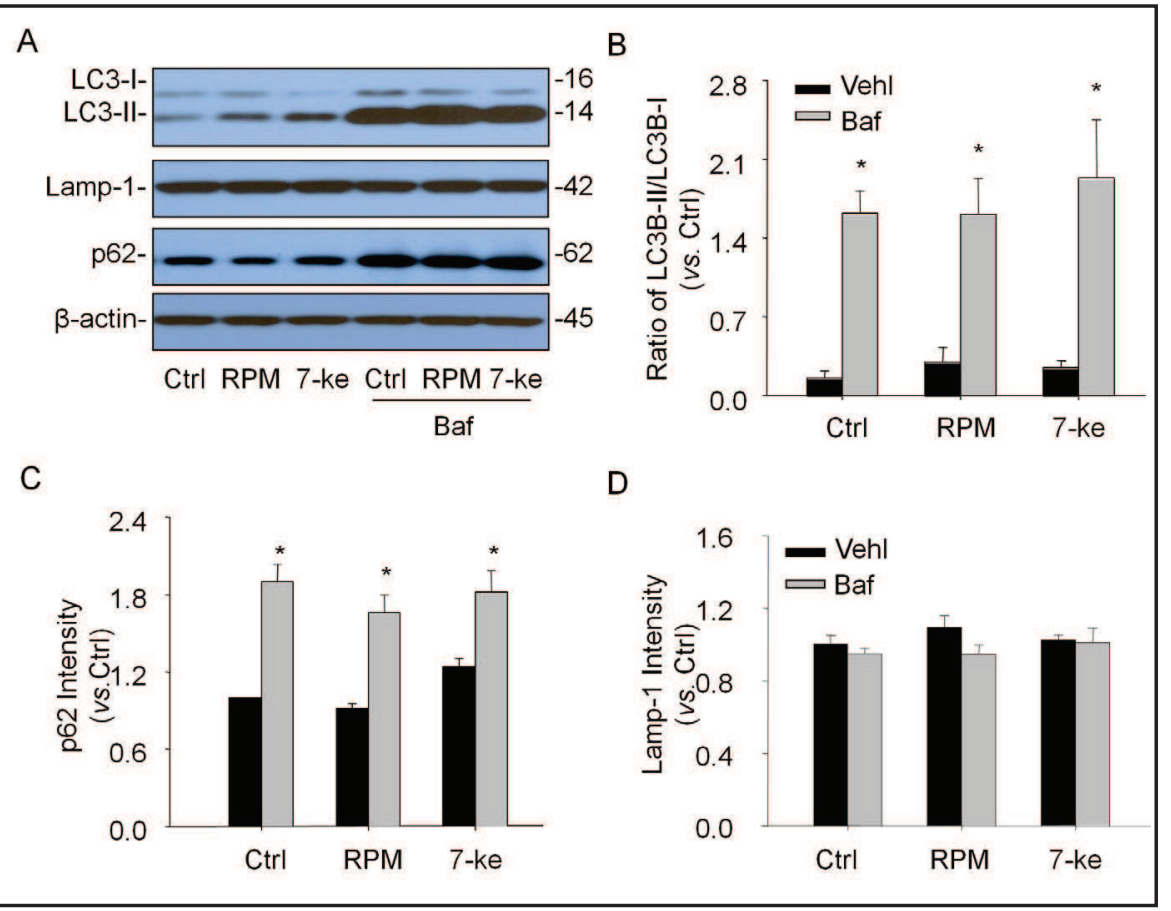
in different groups. B. Summarized data showing expression of LC3B-I and LC3B-II, quantitated as a ratio of LC3B-II band over LC3B-I band ( $n=4)$. C. Summarized data showing expression of p62, quantitated as a ratio of detected specific protein band vs. $\beta$-actin as loading control $(n=4)$. D. Summarized data showing expression of Lamp-1, quantitated as a ratio of detected specific protein band vs. $\beta$-actin as loading control $(n=4)$. * $\mathrm{P}<0.05$ vs. Ctrl: Control, Baf: Bafilomycin, RPM: Rapamycin, 7-Keto: 7-Ketocholesterol.

increased by bafilomycin. However, this increase in bafilomycin-induced autophagosome accumulation was significantly attenuated by Sp-1. Moreover, bafilomycin-induced p62 accumulation was markedly inhibited by Sp-1 as shown in Western gel document (Fig. 4C). Densitometric analysis showed that lysosome function inhibition by bafilomycin led to a significant accumulation of p62 in podocytes, which was remarkably lessened by Sp-1 (Fig. 4D). As shown in Fig. 4E, interestingly, enhanced EMT by bafilomycin as shown by decrease in $\mathrm{P}$-cad and increase in $\alpha$-SMA was obviously attenuated by Sp-1. The ratio of P-cad to $\alpha$-SMA, an EMT index, significantly decreased in bafolimycin-treated podocytes. In the presence of Sp-1, P-cadherin to $\alpha$-SMA ratio was significantly reduced (Fig. 4F).

Failure of Nrf2 gene silencing to alter EMT enhanced by lysosome function inhibition

Mechanistically, we first tested the possibility of p62-regulated Nrf2 signaling pathway to participate in the enhancement of EMT by lysosome function inhibition. Fig. 5A shows representative Western blot gel documents illustrating the changes in P-cad, $\alpha$-SMA and p62 induced by bafilomycin before and after Nrf2 gene silencing (siNrf). The level of P-cad was markedly decreased, while p62 increased in bafilomycin-treated podocytes. These bafilomycin-induced changes in EMT markers in associated with increase in p62 were not altered by Nrf2 gene silencing. As shown in Fig. 5B, bafilomycin significantly decreased the ratio of P-cad and $\alpha$-SMA, an EMT index. However, Nrf2 gene silencing did not alter the ratio of P-cad to $\alpha$-SMA. In addition, bafilomycin-induced significant accumulation of p62 was also not altered by Nrf2 siRNA (Fig. 5C).

No changes in enhanced EMT by inhibition of NF- $\kappa B$-mediated transcriptional regulation

Given the role of NF- $\mathrm{BB}$-mediated transcriptional regulation in cell dedifferentiation and its association with p62, we tested whether inhibition of its activity alters podocyte 


\section{Cellular Physiology Cell Physiol Biochem 2015;35:1773-1786

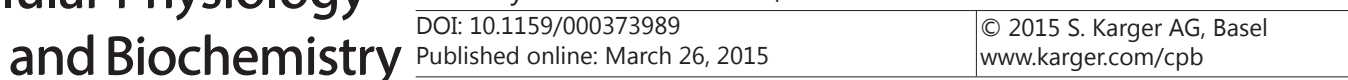

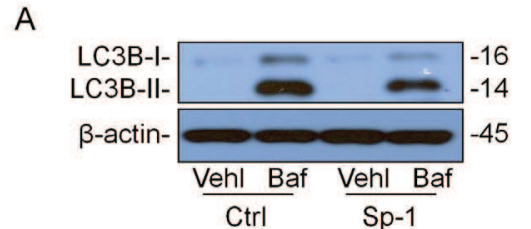

B

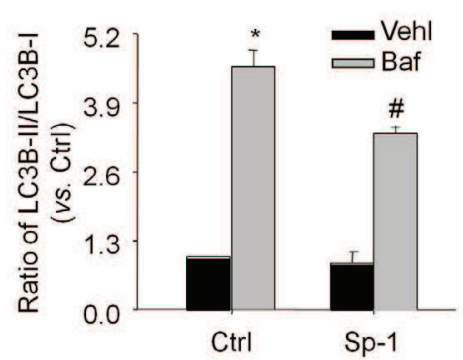

E

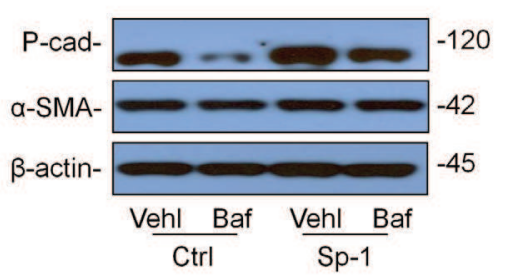

C

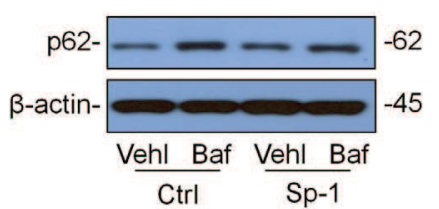

D

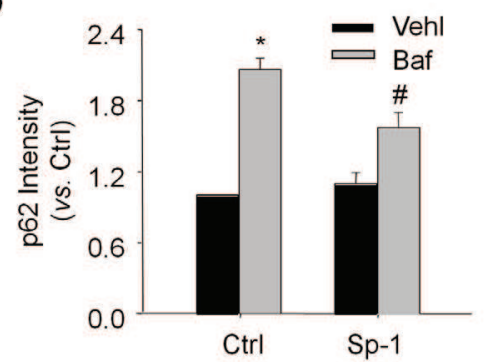

$\mathrm{F}$

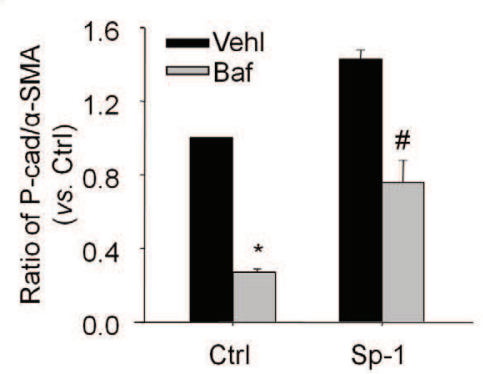

Fig. 4. Sp-1 inhibition of autophagosome formation during lysosome function inhibition. A. Representative gel documents showing the expression of LC3B-I and LC3B-II as autophagosome markers in different groups. B. Summarized data showing expression of LC3B-I and LC3B-II, quantitated as a ratio of LC3B-II band over LC3B-I band (n=3). C. Representative gel documents showing the expression of p62 as autophagosome marker in different groups. D. Summarized data showing expression of p62, quantitated as a ratio of detected specific protein band vs. $\beta$-actin as loading control $(n=3)$. E. Representative gel documents showing the expression of P-cadherin, $\alpha$-SMA in different groups. F. Summarized data showing expression of P-cadherin and $\alpha$-SMA, quantitated as a ratio of P-cadherin band over $\alpha$-SMA band ( $=3-4)$. ${ }^{*} \mathrm{P}<0.05$ vs. Ctrl; \# $\mathrm{P}<0.05$ vs. bafilomycin. Ctrl: Control, Baf: Bafilomycin, Vehl: Vehicle.

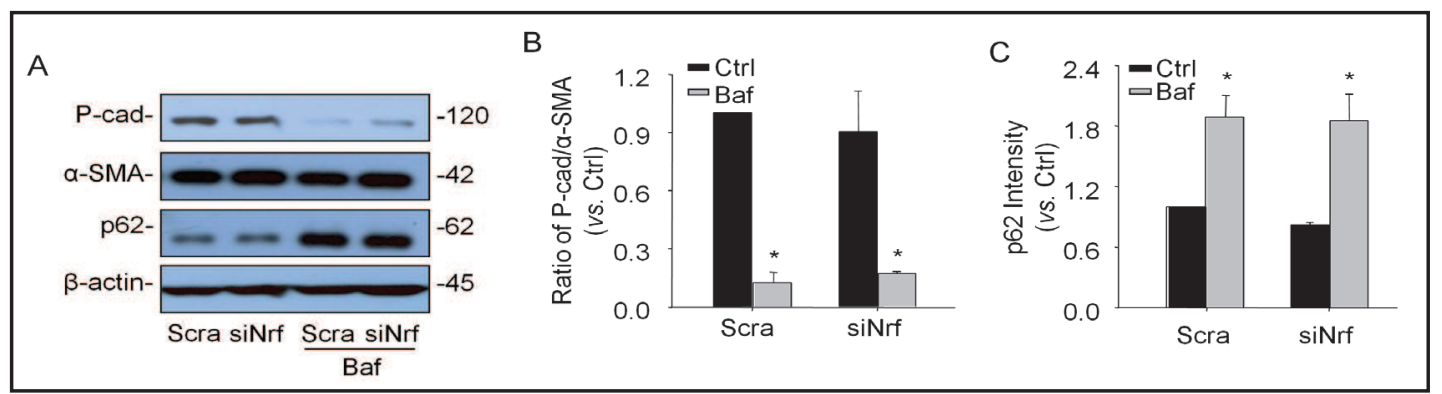

Fig. 5. Failure of Nrf2 inhibition by gene silencing to alter EMT enhanced by lysosome function inhibition. A. Representative gel documents showing the expression of P-cadherin, $\alpha$-SMA and p62 in different groups. B. Summarized data showing expression of P-cadherin and $\alpha$-SMA, quantitated as a ratio of P-cadherin band over $\alpha$-SMA band ( $n=3-4)$. C. Summarized data showing expression of p62, quantitated as a ratio of detected specific protein band vs. $\beta$-actin as loading control ( $n=3-4)$. * $\mathrm{P}<0.05$ vs. Ctrl. Ctrl: Control, Baf: Bafilomycin, Scra: Scramble.

EMT enhanced by lysosome function inhibition. Representative Western blot gel documents in Fig. 6A showed that the level of P-cad remarkably decreased, but p62 was increased in 


\section{Cellular Physiology Cell Physiol Biochem 2015;35:1773-1786

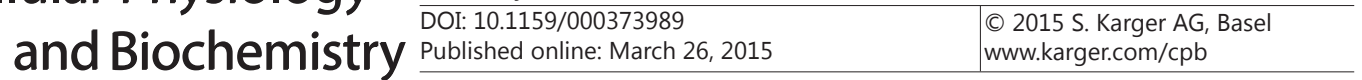
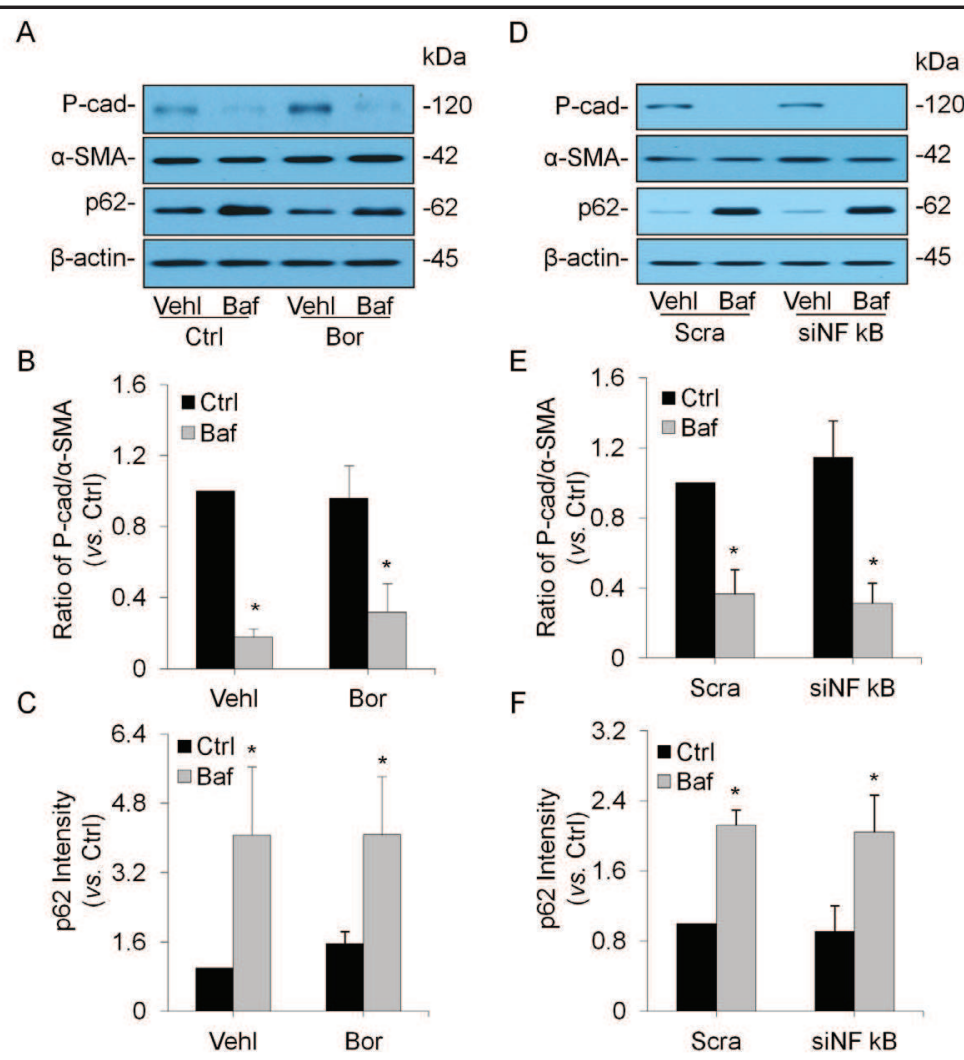

Fig. 6. No changes in enhanced EMT by inhibition of NF-кB-mediated transcriptional regulation. A. Representative gel documents showing the expression of P-cadherin, $\alpha$-SMA and p62 in different groups. B. Summarized data showing expression of P-cadherin and $\alpha$-SMA, quantitated as a ratio of P-cadherin band over $\alpha$-SMA band $(n=5)$. C. Summarized data showing expression of $\mathrm{p} 62$, quantitated as a ratio of detected specific protein band vs. $\beta$-actin as loading control $(n=5)$. D. Representative gel documents showing the expression of P-cadherin, $\alpha$-SMA and p62 in different groups. E. Summarized data showing expression of P-cadherin and $\alpha$-SMA, quantitated as a ratio of P-cadherin band over $\alpha$-SMA band $(n=5)$. F. Summarized data showing expression of p62, quantitated as a ratio of detected specific protein band vs. $\beta$-actin as loading control $(\mathrm{n}=4) .{ }^{*} \mathrm{P}<0.05$ vs. Ctrl. Ctrl: Control, Baf: Bafilomycin, Scra: Scramble.

bafilomycin-treated podocytes. These bafilomycin-induced changes in EMT markers as well as p62 were same before and after pharmacological inhibition of NF- $\mathrm{kB}$ activity. As summarized in Fig. 6B, bafilomycin significantly decreased the ratio of P-cad vs. $\alpha$-SMA, the EMT index, which was not altered by inhibition of NF- $\kappa B$ activity. Furthermore, bafilomycininduced significant accumulation of p62 remained same even though NF- $\kappa B$ activity was inhibited (Fig. 6C). We also inhibited NF-кB-mediated transcriptional regulation by gene silencing to further confirm whether this transcription factor is involved in bafilomycinenhanced EMT. As shown in Fig. 6D, 6E and 6F, the effect of NF- $\kappa B$ gene silencing (siNF-kB) on bafilomycin-enhanced EMT was similar to its pharmacological inhibition, without effects on decreases in the ratio of P-cad vs. $\alpha$-SMA and p62 accumulation in podocytes.

Effects of CDK1 inhibition on EMT enhancement induced by lysosome dysfunction

Another signaling pathway being tested is whether inhibition of CDK1 activity and expression alters bafilomycin-induced enhancement of EMT in podocytes. It has been reported that phosphorylation of p62 regulates exit from cell cycle or cell arrest during cell mitosis and that reduced phosphorylation of p62 leads to a faster exit from cell mitosis, controlling cell dedifferentiation and tumorogenesis [20]. In Fig. 7, panel A shows representative Western blot gel documents, depicting that the level of P-cad remarkably decreased, but 


\section{Cellular Physiology Cell Physiol Biochem 2015;35:1773-1786

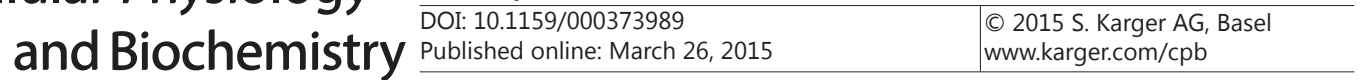
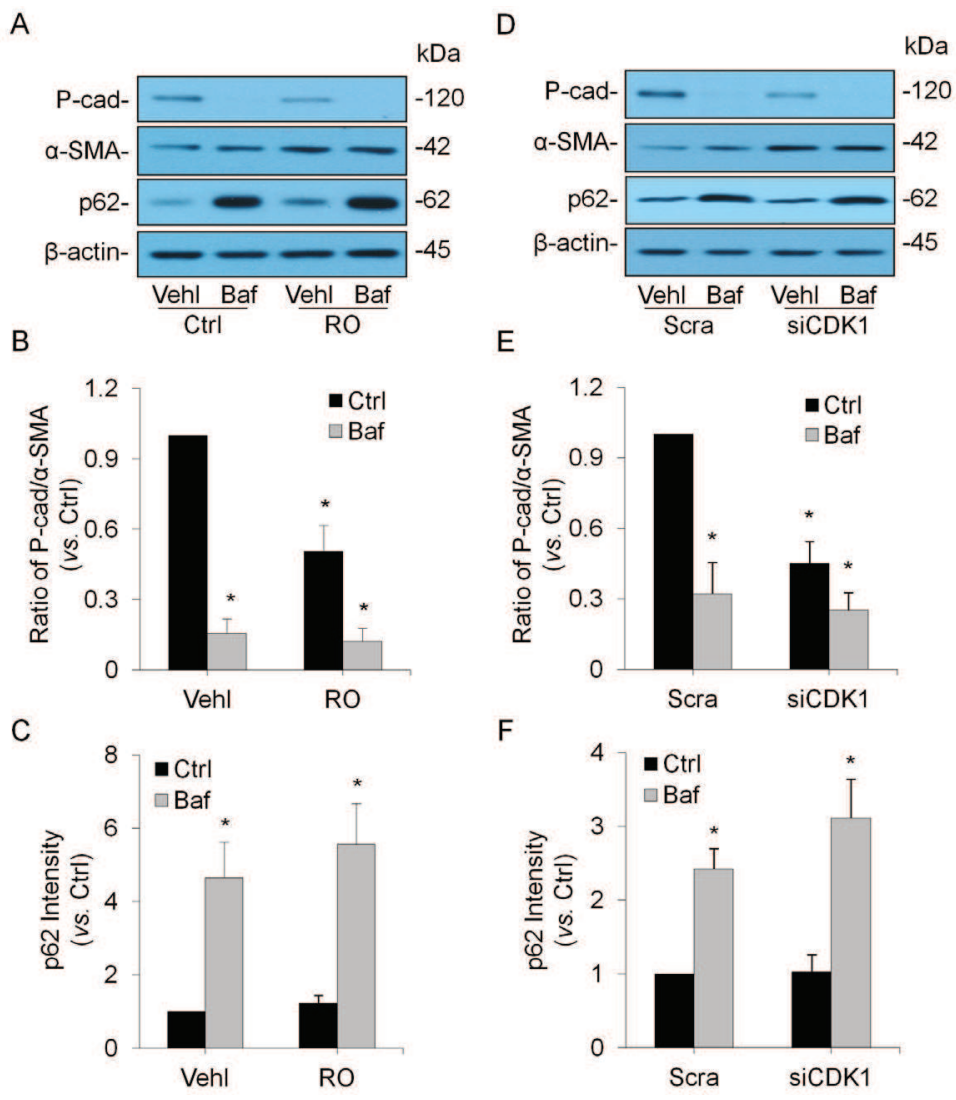

Fig. 7. Effects of CDK1 inhibition on EMT enhancement induced by lysosome dysfunction. A. Representative gel documents showing the expression of P-cadherin, $\alpha$-SMA and p62 in different groups. B. Summarized data showing expression of P-cadherin and $\alpha$-SMA, quantitated as a ratio of P-cadherin band over $\alpha$-SMA band $(n=5)$. C. Summarized data showing expression of p62, quantitated as a ratio of detected specific protein band vs. $\beta$-actin as loading control $(n=9) .{ }^{*} \mathrm{P}<0.05$ vs. Ctrl. D. Representative gel documents showing the expression of P-cadherin, $\alpha$-SMA and p62 in different groups. E. Summarized data showing expression of P-cadherin and $\alpha$-SMA, quantitated as a ratio of P-cadherin band over $\alpha$-SMA band $(n=5)$. F. Summarized data showing expression of p62, quantitated as a ratio of detected specific protein band vs. $\beta$-actin as loading control (n=7). * $\mathrm{P}<0.05$ vs. Ctrl. Ctrl: Control, Baf: Bafilomycin, Scra: Scramble.

$\alpha$-SMA and p62 increased in bafilomycin-treated podocytes. Similar to bafilomycin, CDK1 inhibitor, a quinolinyl thiazolinone derivative, RO-3306 (RO) also decreased the level of P-cad markedly, but increased $\alpha$-SMA and p62 in podocytes. In the presence of RO, bafilomycininduced changes in EMT markers were almost same as that observed in the absence of RO. However, R0 was without effect on p62 level before and after stimulation of bafilomycin. Fig. 7B presents summarized results showing that the ratio of P-cad to $\alpha$-SMA was significantly reduced by both bafilomycin and RO. Moreover, the inhibition of CDK1 by RO had no effect on the p62 level in podocytes under control condition and upon bafilomycin treatment (Fig. 7C). To further confirm the role of CDK1 in enhancement of EMT, we used its siRNA to test whether gene silencing of CDK1 alters EMT. As shown in Fig. 7D, 7E and 7F, CDK1 siRNA (siCDK1) had similar effects on EMT to CDK1 inhibitor-RO, enhancing EMT under control condition without further influence on bafolimycin-induced enhancement of EMT. It was also without effects on p62 levels in podocytes with or without treatment of bafolimycin.

\section{Reduction of 662 phosphorylation during lysosome function inhibition}

Further experiments were designed to determine whether reduced p62 phosphorylation occurs due to lysosome function inhibition. As shown in Fig. 8A, Western blot gel documents 


\section{Cellular Physiology Cell Physiol Biochem 2015;35:1773-1786

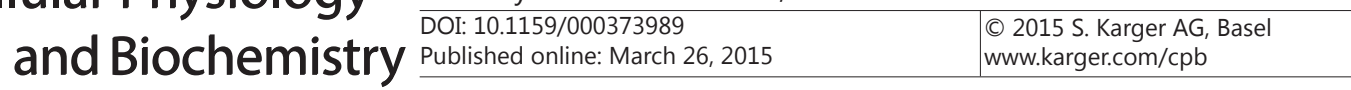

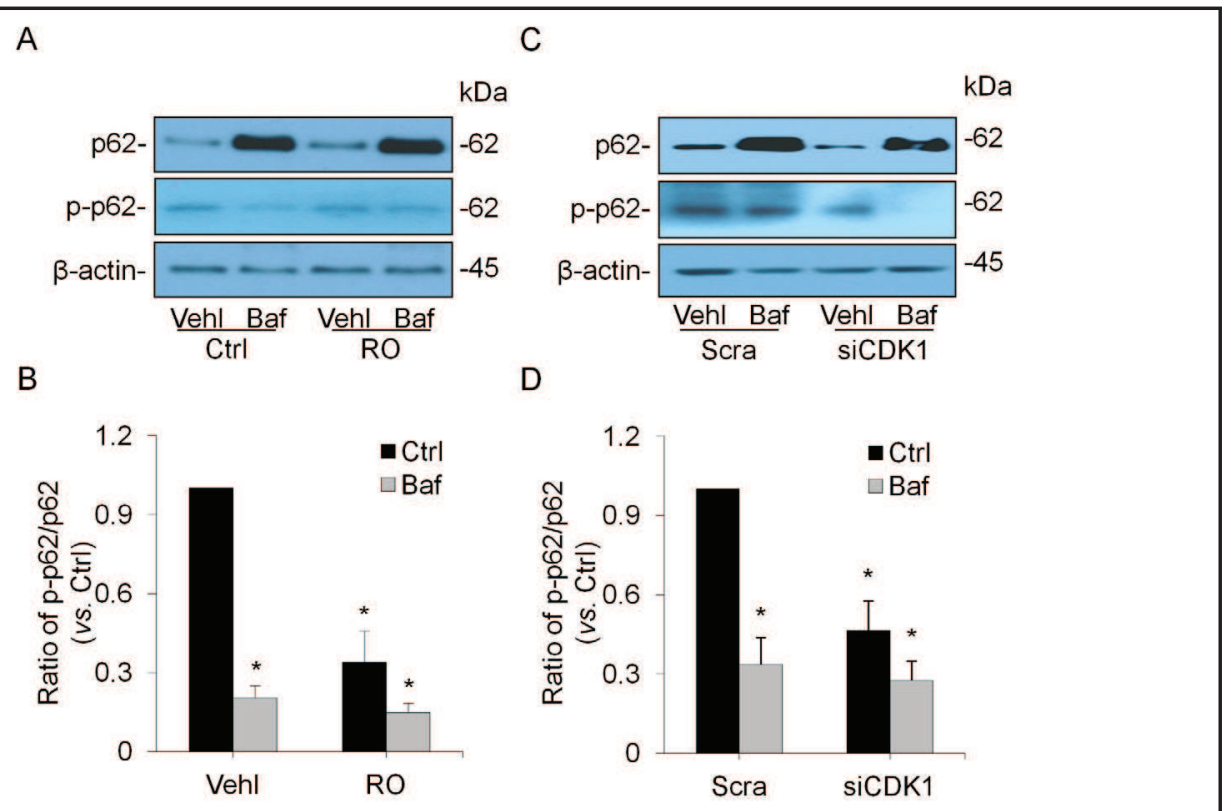

Fig. 8. Reduction of p62 phosphorylation during lysosome function inhibition. A. Representative gel documents showing the expression of p62 and p-p62 in different groups. B. Summarized data showing expression of p62 and p-p62, quantitated as a ratio of p-p62 band over p62 band (n=5). C. Representative gel documents showing the expression of p62 and p-p62 in different groups. D. Summarized data showing expression of p62 and p-p62, quantitated as a ratio of p-p62 band over p62 band (n=4). * $\mathrm{P}<0.05$ vs. Ctrl. Ctrl: Control, Baf: Bafilomycin, Scra: Scramble.

show that CDK1 inhbitor RO markedly decreased phosphorylated p62 even though it had no effect on total p62 level in podocytes. However, bafilomycin mainly led to p62 accumulation, but it had no effect on phosphorylation of p62. By calculation, the ratio of phosphorylated p62 to total p62 was found reduced significantly by both R0 and bafilomycin, suggesting that the relative reduction of phosphorylated p62 may be involved in the control of EMT (Fig. 8B). We also conducted additional experiments to silence CDK1 gene in order to confirm the role of decreased phophorylated p62 in podocyte EMT. As presented in Fig. 8C, similar to RO, CDK1 siRNA reduced phophorylated p62, but had no effects on total p62 level. As summarized in Fig. 8D, this CDK1 siRNA reduced the ratio of phosphorylated p62 to total p62, which was similar to the effects of bafilomycin.

\section{Discussion}

The major goals of the present study were to determine whether lysosome dysfunctioninduced podocyte EMT is attributed to the accumulation of autophagosome and p62, which are degraded by autophagic process and to explore the mechanisms by which accumulation of p62 exerts its action as a signaling hub to activate or enhance EMT in podocytes. It was found that lysosome function inhibition with a low dose of lysosomal V-ATPase inhibitor, Baf A1 (5 nM) or by silencing V-ATPase gene remarkably enhanced podocyte EMT, which was accompanied by autophagosome accumulation due to the deficient autophagic flux. This enhancement of EMT in podocytes was significantly attenuated by inhibition of the autophagosome formation with $\mathrm{Sp}-1$, suggesting that autophagosome accumulation in podocytes contributes to enhanced EMT induced by lysosome dysfunction. We further explored the signaling mechanisms activating or enhancing podocyte EMT associated with lysosome dysfunction. Given that p62 was found significantly increased during lysosome dysfunction and resulting deficiency of autophagic flux, several signaling pathways 


\section{Cellular Physiology Cell Physiol Biochem 2015;35:1773-1786

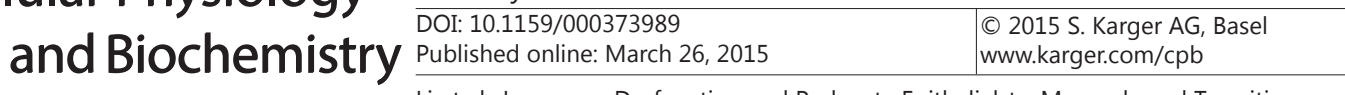 \\ Li et al.: Lysosome Dysfunction and Podocyte Epithelial-to-Mesenchymal Transition}

regulated via p62 were tested including Nrf2-mediated redox signaling, NF- $\kappa B$-dependent transcriptional regulation, and CDK1-mediated phosphorylation of p62 as an intracellular controller of cell mitosis exit. Our results indicate that reduction of CDK1-mediated p62 phosphorylation and consequent speeding exit of cell mitosis are a critical molecular mechanism mediating podocyte EMT during lysosome dysfunction.

It has been reported that podocytes in glomeruli of mammalian animals are highly differentiated and therefore the maintenance of functional and structural integrity of these cells during their long time surviving are much dependent upon the autophagic process, which includes the formation and degradation of autophagosomes [13]. This led us to hypothesize that lysosome dysfunction and consequent derangement of autophagic flux may be an important mechanism activating or enhancing EMT in podocytes, leading to podocyte injury and glomerular sclerosis. This hypothesis was tested in the present study by inhibition of lysosome function using V-ATPase inhibitor, bafilomycin A1 (Baf A1) and specific V-ATPase siRNA. We indeed demonstrated that inhibition of lysosome function by Baf A1 and V-ATPase siRNA significantly enhanced EMT in podocytes, which was comparable to that produced by a well-established EMT inducer, TGF- $\beta[17,18]$ or by deletion or knocking down of CD38, an enzyme for production of an endogenous regulator of lysosome function, NAADP $[13,16]$. To our knowledge, these results represent the first experimental evidence that inhibition of lysosomal V-ATPase activity or its gene silencing activated or enhanced podocyte EMT. In previous studies, some molecular mechanisms have been proposed to activate EMT in other cell or tissue types, which are similar to those regulating oncogenic properties in neoplastic cells such as proliferation, resistance to apoptosis and angiogenesis through transcription factors [4]. However, these mechanisms are found mainly related to ubiquitination, namely, the covalent link of the small 76-amino acid protein ubiquitin to target proteins, which serves as a signal for many proteins to be degraded by the proteasomes [4]. Although lysosomal degradation of some signaling proteins may be one of mechanisms responsible for EMT, little is known so far how lysosome dysfunction induces EMT in many cell types including podocytes. In this regard, some previous studies demonstrated that Raf and TGF- $\beta$ may work together to promote lysosomal degradation of E-cadherin instead of its recycling in tumor cells, resulting in EMT and tumor progression [19]. Despite these observations of lysosomal degradation of signaling proteins in EMT, it remains unknown how lysosomal degradation of signaling proteins activates EMT. In particular, it is interesting to know whether the reversal of differentiation induced by autophagy, a process fine controlled by lysosome function, contributes to EMT in podocytes. If so, we need to test what is the triggering mechanism.

We first examined whether enhanced EMT during lysosome dysfunction is associated with deficient autophagy, in particular, the autophagic flux, given the important role of lysosomes in this process. It was demonstrated that autophagosome and p62, a scaffold protein also in autophagic process, were largely accumulated in podocytes by Baf A1 or silencing of v-ATPase gene, suggesting that lysosome function inhibition leads to abnormal autophagic flux and thereby reduces autophagic degradation of ubiquitinated cargoes and functional substrates involved in the autophagic process such as p62 [20-23]. We also demonstrated that inhibition of autophagosome formation by $\mathrm{Sp}-1$ significantly attenuated the enhancement of EMT induced by lysosome dysfunction induced by Baf A1 and V-ATPase siRNA. To our knowledge, these results provide the first evidence that autophagosome accumulation may serve as a critical mechanism activating or enhancing EMT in podocytes. In some previous studies, autophagy was shown to be critical for the invasion of tumor cells, which is associated with the induction of EMT and activation of TGF- $\beta /$ Smad3-dependent signaling pathway [24]. In addition, the autophagy process and the autophagy-mediated lysosomal degradation of SNAI/Snail and TWIST, two master inducers of the EMT process have been reported to mediate the effects of death effector domain-containing DNA-binding protein (DEDD) to alter tumor growth and metastasis, suggesting that autophagy is involved in EMT and subsequent growth or metastasis of tumors [25, 26].

After confirmation of deficient autophagic flux as an important process, we went on to address how Baf A1-induced accumulation of autophagosome initiated EMT in podocytes. 


\section{Cellular Physiology Cell Physiol Biochem 2015;35:1773-1786

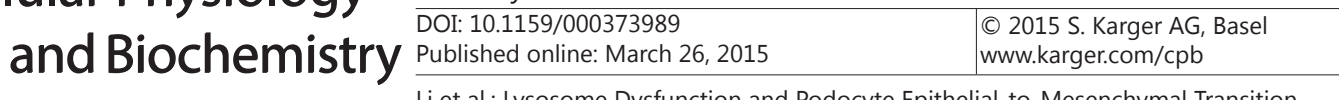

It has been reported that p62 as a signaling hub regulates cell proliferation and many other activities $[27,28]$ and that in tumor cells, p62 is required for cell transformation $[27,29$, $30]$. Interestingly, the genetic inactivation of key autophagy molecules, such as Atg7, results in p62 accumulation and hepatotoxicity, which leads to the generation of liver tumors [27, $29]$, suggesting the important association of autophagic process of p62 with tumorogenesis or cell transdifferentiation. We tested the role of 3 pathways regulated p62 in podocyte EMT, which include NF- $\kappa B$, Nrf2 and cyclin-dependent kinases (CDKs). Using selective inhibitor or siRNAs, we found that inhibition of NF- $\mathrm{KB}$ and Nrf2 signaling pathways had no effect on Baf A1-induced EMT in podocytes, suggesting that both pathways are not involved in this podocytes transdifferentiation induced by lysosome dysfunction. However, inhibition of CDK1 activity or silencing its gene produced podocyte EMT without effect on the level of p62. In the presence of CDK1 inhibitor or siRNA, Baf-induced podocyte EMT was markedly attenuated by $60 \%$, which was accompanied by significantly reduced phophosrylation of $\mathrm{p} 62$ in these podocytes. These results suggest that CDK1 phosphorylation exerts an important regulatory role on podocyte EMT and that reduced CDK1 phosphorylation may result in enhancement of this EMT. To our knowledge, there have been no reports on the role of CDK1-mediated p62 phosphorylation in the regulation of podocytes differentiation or transdifferentiation. Our results provide direct evidence that this CDK1-mediated mechanism critically contributes to EMT activation. In studies using other cell types, CDKs were dermonstrated to regulate the progression of mammalian cells through the various phases of the cell cycle [31]. Among these CDKs, CDK1 controls transit through the late S/G2 phase and early mitosis phase of the cell cycle [32,33], which may be associated with p62 phosphorylation at residues T269 and S272 [34, 35]. In cancer cells, expression of a nonphosphorylatable p62 mutant displayed higher tumorigenic properties than the same cells expressing wild-type p62 [35]. Moreover, p62 phosphorylation has been shown to play an important role in the stabilization of cyclin B1, which interacts with CDK1 to specifically regulate the entry into mitosis [35]. Our findings together with these previous results provide strong evidence that reduced phosphorylation of p62 due to inhibition of CDK1 during lysosome dysfunction leads to a faster exit from cell mitosis and thereby enhances EMT in podocytes, which is resulted from accumulation of p62 induced by deranged autophagic flux.

In summary, the present study revealed a new triggering mechanism of podocyte EMT during lysosome dysfunction, which is characterized by deranged autophagic flux, p62 accumulation and associated reduction of p62 phosphorylation. This dysregulation of p62 and its CDK1-dependent phosphorylation may represent a novel early event leading to podocyte dysfunction and injury, which may initiate podocytes injury and ultimately result in glomerulosclerosis during lysosome dysfunction. These results may direct toward the development of new therapeutic strategies targeting phosphorylation of p62 for prevention or treatment of glomerular sclerosis associated with lysosome dysfunction and deficient autophagy under different pathological conditions such as hypercholesterimia, hyperhomocysteinemia or diabetes mellitus.

\section{Acknowledgements}

This study was supported by grants DK54927, HL75316 and HL57244 from National Institutes of Health.

\section{Disclosure Statement}

No competing financial interests exist. 


\section{Cellular Physiology Cell Physiol Biochem 2015;35:1773-1786 \begin{tabular}{ll|l} 
and Biochemistry & $\begin{array}{l}\text { DOI: 10.1159/000373989 } \\
\text { Publisned onme: Ivirch 26, } 2015\end{array}$ & $\begin{array}{l}\text { C 2015 S. Karger AG, Basel } \\
\text { www.karger.com/cpb }\end{array}$ \\
\hline
\end{tabular}}

Li et al.: Lysosome Dysfunction and Podocyte Epithelial-to-Mesenchymal Transition

\section{References}

1 Anil Kumar P, Welsh GI, Saleem MA, Menon RK: Molecular and cellular events mediating glomerular podocyte dysfunction and depletion in diabetes mellitus. Front Endocrinol (Lausanne) 2014;5:151.

2 Asanuma K, Mundel P: The role of podocytes in glomerular pathobiology. Clin Exp Nephrol 2003;7:255259.

3 Menini S, Iacobini C, Oddi G, Ricci C, Simonelli P, Fallucca S, Grattarola M, Pugliese F, Pesce C, Pugliese G: Increased glomerular cell (podocyte) apoptosis in rats with streptozotocin-induced diabetes mellitus: Role in the development of diabetic glomerular disease. Diabetologia 2007;50:2591-2599.

4 Kang YS, Li Y, Dai C, Kiss LP, Wu C, Liu Y: Inhibition of integrin-linked kinase blocks podocyte epithelialmesenchymal transition and ameliorates proteinuria. Kidney Int 2010;78:363-373.

-5 Sam R, Wanna L, Gudehithlu KP, Garber SL, Dunea G, Arruda JA, Singh AK: Glomerular epithelial cells transform to myofibroblasts: Early but not late removal of tgf-beta1 reverses transformation. Transl Res 2006;148:142-148.

6 Guo J, Xia N, Yang L, Zhou S, Zhang Q, Qiao Y, Liu Z: Gsk-3beta and vitamin d receptor are involved in betacatenin and snail signaling in high glucose-induced epithelial-mesenchymal transition of mouse podocytes. Cell Physiol Biochem 2014;33:1087-1096.

7 Li CX, Xia M, Han WQ, Li XX, Zhang C, Boini KM, Liu XC, Li PL: Reversal by growth hormone of homocysteine-induced epithelial-to-mesenchymal transition through membrane raft-redox signaling in podocytes. Cell Physiol Biochem 2011;27:691-702.

-8 Xia M, Conley SM, Li G, Li PL, Boini KM: Inhibition of hyperhomocysteinemia-induced inflammasome activation and glomerular sclerosis by nlrp3 gene deletion. Cell Physiol Biochem 2014;34:829-841.

-9 Yi F, Li PL: Mechanisms of homocysteine-induced glomerular injury and sclerosis. Am J Nephrol 2008;28:254-264.

10 Yi F, Xia M, Li N, Zhang C, Tang L, Li PL: Contribution of guanine nucleotide exchange factor vav2 to hyperhomocysteinemic glomerulosclerosis in rats. Hypertension 2009;53:90-96.

-11 Zhang C, Hu JJ, Xia M, Boini KM, Brimson C, Li PL: Redox signaling via lipid raft clustering in homocysteineinduced injury of podocytes. Biochim Biophys Acta 2010;1803:482-491.

12 Levine B, Kroemer G: Autophagy in the pathogenesis of disease. Cell 2008;132:27-42.

13 Xiong J, Xia M, Xu M, Zhang Y, Abais JM, Li G, Riebling CR, Ritter JK, Boini KM, Li PL: Autophagy maturation associated with cd38-mediated regulation of lysosome function in mouse glomerular podocytes. J Cell Mol Med 2013;17:1598-1607.

$\checkmark 14$ Gozuacik D, Kimchi A: Autophagy as a cell death and tumor suppressor mechanism. Oncogene 2004;23:2891-2906.

-15 Marino G, Fernandez AF, Cabrera S, Lundberg YW, Cabanillas R, Rodriguez F, Salvador-Montoliu N, Vega JA, Germana A, Fueyo A, Freije JM, Lopez-Otin C: Autophagy is essential for mouse sense of balance. J Clin Invest 2010;120:2331-2344.

16 Boini KM, Xia M, Xiong J, Li C, Payne LP, Li PL: Implication of cd38 gene in podocyte epithelial-tomesenchymal transition and glomerular sclerosis. J Cell Mol Med 2012;16:1674-1685.

17 Li Y, Kang YS, Dai C, Kiss LP, Wen X, Liu Y: Epithelial-to-mesenchymal transition is a potential pathway leading to podocyte dysfunction and proteinuria. Am J Pathol 2008;172:299-308.

18 Linares JF, Amanchy R, Greis K, Diaz-Meco MT, Moscat J: Phosphorylation of p62 by cdk1 controls the timely transit of cells through mitosis and tumor cell proliferation. Mol Cell Biol 2011;31:105-117.

19 Voutsadakis IA: Ubiquitination and the ubiquitin-proteasome system as regulators of transcription and transcription factors in epithelial mesenchymal transition of cancer. Tumour Biol 2012;33:897-910.

20 Bitto A, Lerner CA, Nacarelli T, Crowe E, Torres C, Sell C: P62/sqstm1 at the interface of aging, autophagy, and disease. Age (Dordr) 2014;36:9626.

-21 Janda E, Nevolo M, Lehmann K, Downward J, Beug H, Grieco M: Raf plus tgfbeta-dependent emt is initiated by endocytosis and lysosomal degradation of e-cadherin. Oncogene 2006;25:7117-7130.

22 Johansen T, Lamark T: Selective autophagy mediated by autophagic adapter proteins. Autophagy 2011;7:279-296.

23 Matsumoto G, Wada K, Okuno M, Kurosawa M, Nukina N: Serine 403 phosphorylation of p62/sqstm1 regulates selective autophagic clearance of ubiquitinated proteins. Mol Cell 2011;44:279-289. 


\section{Cellular Physiology Cell Physiol Biochem 2015;35:1773-1786 \begin{tabular}{ll|l} 
and Biochemistry & $\begin{array}{l}\text { DOI: 10.1159/000373989 } \\
\text { Publisned onine: 1varch 26, } 2015\end{array}$ & $\begin{array}{l}\text { O 2015 S. Karger AG, Basel } \\
\text { www.karger.com/cpb }\end{array}$ \\
\cline { 2 - 3 }
\end{tabular}}

Li et al.: Lysosome Dysfunction and Podocyte Epithelial-to-Mesenchymal Transition

24 Komatsu M, Waguri S, Koike M, Sou YS, Ueno T, Hara T, Mizushima N, Iwata J, Ezaki J, Murata S, Hamazaki J, Nishito Y, Iemura S, Natsume T, Yanagawa T, Uwayama J, Warabi E, Yoshida H, Ishii T, Kobayashi A, Yamamoto M, Yue Z, Uchiyama Y, Kominami E, Tanaka K: Homeostatic levels of p62 control cytoplasmic inclusion body formation in autophagy-deficient mice. Cell 2007;131:1149-1163.

25 Li J, Yang B, Zhou Q Wu Y, Shang D, Guo Y, Song Z, Zheng Q, Xiong J: Autophagy promotes hepatocellular carcinoma cell invasion through activation of epithelial-mesenchymal transition. Carcinogenesis 2013;34:1343-1351.

-26 Lv Q Hua F, Hu ZW: Dedd, a novel tumor repressor, reverses epithelial-mesenchymal transition by activating selective autophagy. Autophagy 2012;8:1675-1676.

27 Lv Q Wang W, Xue J, Hua F, Mu R, Lin H, Yan J, Lv X, Chen X, Hu ZW: Dedd interacts with pi3kc3 to activate autophagy and attenuate epithelial-mesenchymal transition in human breast cancer. Cancer Res 2012;72:3238-3250.

-28 Moscat J, Diaz-Meco MT: P62: A versatile multitasker takes on cancer. Trends Biochem Sci 2012;37:230236.

29 Moscat J, Diaz-Meco MT: P62 at the crossroads of autophagy, apoptosis, and cancer. Cell 2009;137:10011004.

30 Nezis IP, Stenmark H: P62 at the interface of autophagy, oxidative stress signaling, and cancer. Antioxid Redox Signal 2012;17:786-793.

-31 Inami Y, Waguri S, Sakamoto A, Kouno T, Nakada K, Hino O, Watanabe S, Ando J, Iwadate M, Yamamoto M, Lee MS, Tanaka K, Komatsu M: Persistent activation of nrf2 through p62 in hepatocellular carcinoma cells. J Cell Biol 2011;193:275-284.

32 Malumbres M, Barbacid M: Mammalian cyclin-dependent kinases. Trends Biochem Sci 2005;30:630-641.

-33 Nigg EA: Mitotic kinases as regulators of cell division and its checkpoints. Nat Rev Mol Cell Biol 2001;2:2132.

-34 Wang Z, Fan M, Candas D, Zhang TQ Qin L, Eldridge A, Wachsmann-Hogiu S, Ahmed KM, Chromy BA, Nantajit D, Duru N, He F, Chen M, Finkel T, Weinstein LS, Li JJ: Cyclin b1/cdk1 coordinates mitochondrial respiration for cell-cycle g2/m progression. Dev Cell 2014;29:217-232.

-35 Ye X, Sloboda RD: Molecular characterization of p62, a mitotic apparatus protein required for mitotic progression. J Biol Chem 1997;272:3606-3614. 